\title{
Seawater motion-induced electromagnetic noise reduction in marine magnetotelluric data using current meters
}

\author{
Kai Chen ${ }^{1 *}$, Qingxian Zhao ${ }^{2}$, Ming Deng ${ }^{1}$, Xianhu Luo ${ }^{2}$ and Jianen Jing ${ }^{1}$
}

\begin{abstract}
Seawater motion-induced electromagnetic (EM) noise along the seafloor has a large impact on marine magnetotelluric (MT) data quality. Although the mechanical stability of ocean bottom electromagnetic receivers (OBEMs) has improved due to buoyancy optimization, completely eliminating EM noise generated by seafloor currents as a result of instrument rocking or induction from the Earth's magnetic field is still not possible. The velocity of the current represents the quantification of seafloor conditions. To mitigate this problem, we installed a current meter on an OBEM to measure the synchronous current velocity along with the OBEM data logger. For the marine EM surveys, we conducted two surveys composed of 42 marine EM data acquisition sites in the South China Sea. We observed a strong correlation between induced EM noise and current velocity when the speed was greater than $2 \mathrm{~cm} / \mathrm{s}$. Furthermore, we developed an adaptive correlation noise-canceling filter to reduce the induced EM noise, using the current meter data as a reference signal. The filter refined the coefficients using a least-mean-squares algorithm. We were able to reduce the induced EM noise by pre-filtering the raw time series data with an adaptive correlation noise-canceling filter and using current meter data from nearby sites. Since seafloor currents are clearly an issue that limits MT data quality, special efforts are necessary to reduce seawater motion-induced EM noise in marine MT surveys.
\end{abstract}

Keywords: Marine magnetotelluric, Adaptive correlation noise-canceling filter, Seawater motion-induced electromagnetic noise, Current meter

\section{Introduction}

Since the 1970s, a wide range of electromagnetic (EM) methods, such as the magnetotelluric (MT) method, have been used for deep marine detection (Vozoff 1972) and have proven successful for studying volcanoes (Constable and Heinson 2004), mid-ocean ridges (Baba et al. 2006), subduction zones (Naif et al. 2015), and in petroleum exploration (Key et al. 2006). Marine MT surveys use ocean bottom electromagnetic (OBEM) receivers on the seafloor to measure natural MT source signals (Filloux 1980). Recently, due to advancements in semiconductor and information technology, these instruments now have

\footnotetext{
*Correspondence: ck@cugb.edu.cn

${ }^{1}$ School of Geophysics and Information Technology, China University

of Geosciences, Beijing, China

Full list of author information is available at the end of the article
}

broader bandwidths, lower power consumption, and a lower level of internal sensor noise, especially in the effective bandwidth that is extendable to $0.1 \mathrm{~Hz}$ in deep water (approximately $1000 \mathrm{~m}$ ) (Constable et al. 1998).

While an OBEM is deployed on the seafloor, it records the MT signal, which is attenuated due to highly conductive seawater; internal instrument noise from the electrode, induction coil, and data logger; and EM noise from the environment. EM noise from the environment includes noise from nearby vessels, onshore cultural noise, biological noise, and seawater motion-induced noise. In deep water settings, both vessel and onshore cultural noise are negligible, and there is a low probability of biological noise. EM noise is caused by seawater current EM induction and by rocking of the instrument. It is well known that mechanical motion of an induction coil in the Earth's magnetic field will generate an 
additional magnetic field, and hence correlated noise, but this should not affect the electrical field unless $\mathbf{v} \times \mathbf{B}$ is comparable to the ambient field. Lezaeta et al. (2005) previously showed that some sites display electric as well as magnetic field variations that correlated with tilt, suggesting EM induction due to water motion. Seawater motion-induced EM noise is therefore possibly a large source of noise in deep water. This type of noise not only rocks the instrument, but also generates EM noise that couples with the Earth's magnetic field. The coupling of seawater motion-induced EM noise with the Earth's magnetic field will produce an obvious magnetic field and, hence, correlated EM noise (Sanford, 1971; Chave and Luther, 1990) that affects the electric field $(\mathbf{E}=\mathbf{v} \times \mathbf{B})$. This is comparable to the locally induced ambient magnetic field in the surrounding medium, which occurs in the highly conductive ocean and is subject to various water disturbances.

Previous studies have unsuccessfully attempted to reduce seawater motion-induced EM noise. Key and Constable (2002) used a multi-station process code developed by Egbert (1997) to process marine MT data, which is a robust multivariate errors-in-variables (RMEV) approach. When EM noise is too strong, improvement is limited, and data contaminated by the induced EM noise must be removed. Lezaeta et al. (2005) performed noise corrections on shallow-water EM data affected by instrument motion. They measured tilt data to correct for instrument motion noise. Instrument motion noise contaminates the magnetic file channel and induces EM noise in all channels that contain an E- and B-field channel. Neska et al. (2013) reduced motion noise in marine magnetotelluric data by using tilt records, and applied a standard motion-removal technique as well as a newly developed method to correct for motion-induced noise. The tilt measurement method can be used for instrument motion and is less useful for seawater motioninduced EM noise. Modifications to buoyancy design has increased the mechanical stability of the instrument and decreased instrument motion noise, however induced EM noise is still an issue.

Water movement not only occurs at the sea surface, but also along the seafloor. Correspondingly, seafloor current-induced EM noise exists both in shallow and deep water. To analyze the seafloor currents, we developed an OBEM with a current meter that simultaneously measured current data along with the OBEM data logger. If we can obtain seafloor current speeds, we can reduce induced EM noise by using the current meter data to apply an adaptive correlation noise-canceling filter to the OBEM data.

This paper mainly focuses on (1) the discussion of seawater motion-induced EM noise which affects marine
MT data and (2) the development of a time-domain separation technique for correlated noise, using an adaptive correlation noise-cancellation filter, before estimating the frequency-domain MT apparent resistivity and impedance phase.

\section{Data set}

Between August and September 2018, two gas hydrate exploration surveys were conducted in the Shenhu and Qiongdongnan areas of the northern South China Sea. The objective of these surveys was to characterize the background geology and electrical conductivity structure of the region prior to continued exploration in the area, which is a prospective gas-hydrate area (JianEn et al. 2018). Shenhu is located at the edge of the continental shelf, which produces strong currents, and water depths range between 900 and $1700 \mathrm{~m}$. The Qiongdongnan area is a marine basin at the base of the continental shelf with a water depth of approximately $1750 \mathrm{~m}$. Seawater motion in the basin area is quiet compared to the continental shelf/slope area.

We conducted controlled source electromagnetic (CSEM) and MT surveys using the R.V. "Haiyangsihao". Both surveys utilized our developed instrument, which contains two sets of transmitters (Wang et al. 2017) and 25 OBEMs (Kai et al. 2015). Figure 1 presents a map of the two surveys. In the Shenhu area, the experimental layout, shown in the right inset of Fig. 1, consisted of two lines of 20 OBEM receivers spaced $300 \mathrm{~m}$ apart. During a 5-day deployment, we acquired $0.5 \mathrm{~Hz}, 1.5 \mathrm{~Hz}$, and $8 \mathrm{~Hz}$ frequency CSEM data and performed continuous MT data collection. In the Qiongdongnan area, shown in the left inset of Fig. 1, we used 22 OBEMs during a 6-day deployment with $500 \mathrm{~m}$ spacing, as well as collecting additional $0.125 \mathrm{~Hz}$ frequency CSEM data during towing. The transmitter dipole was flown approximately $50 \mathrm{~m}$ above the seafloor and transmitted a 450 A square wave using a 300-m dipole antenna. We recovered all 42 OBEM units after each survey was completed.

An OBEM contains five channels capable of measuring three orthogonal E-field components and two horizontal B-field components. The length of the horizontal E-field component dipole is $12 \mathrm{~m}$ and the length of the vertical $\mathrm{E}$ dipole is $2 \mathrm{~m}$. We installed an additional weight at the tail of the dipole near the $\mathrm{Ag}-\mathrm{AgCl}$ electrode to reduce mechanical motion due to seawater motion. We also upgraded the anchor to increase its weight in water and stabilize the induction coil and electrode arm. Figure 2 shows the OBEM before recovery. The sampling rate was set to $150 \mathrm{~Hz}$ continuous acquisition once the power was turned on. While the OBEM was deployed on the seafloor, the data logger recorded the 3 -axis attitude (yaw, pitch, and roll) once 


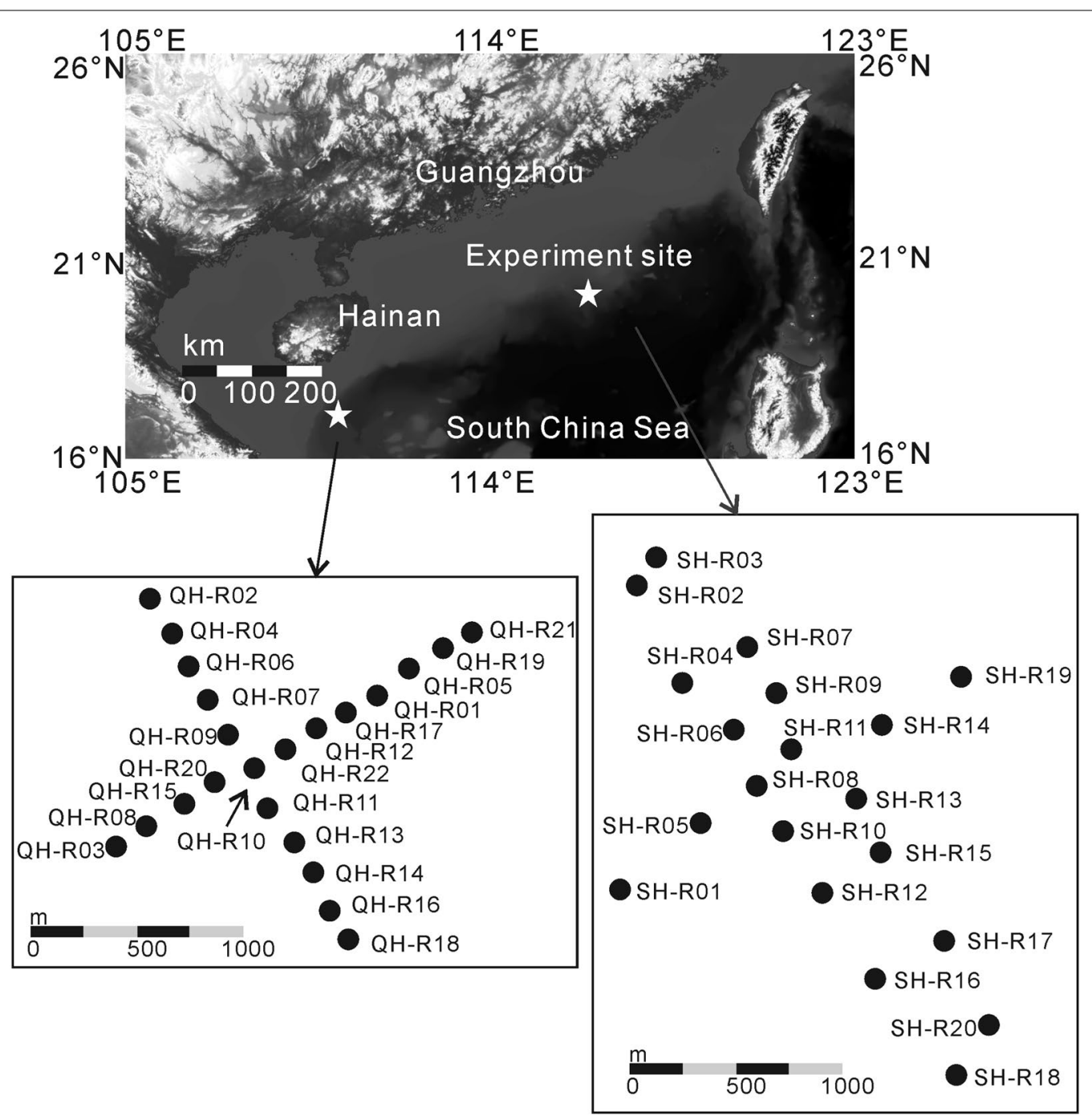

Fig. 1 Locations of marine CSEM and MT stations deployed in the Qiongdongnan (left) and Shenhu (right) areas of the South China Sea. The top panel shows a partial regional map of the South China Sea

per minute. We also installed a current meter on the top of the vertical E dipole. During deployment on the seafloor, the current meter was positively buoyant and was allowed to tilt freely. We used a TCM-3 Tilt (LOWELL Instruments LLC) current meter to record water speed. This unit is designed for use beyond the edge of the continental shelf at depths of up to $4500 \mathrm{~m}$. Tilt current meters measure the current using the dragtilt principle. The meter is buoyant and is secured by a flexible tether fixed to an anchor or tripod. When water motion tilts the logger in the direction of flow, a 3-axis accelerometer and magnetometer determine the tilt and bearing. Syntactic foam allows the device to maintain positive buoyancy. The meter has a recommended range of $80 \mathrm{~cm} / \mathrm{s}$ and measures the velocity in two directions referenced to north and east. We set the sampling rate at $1 \mathrm{~Hz}$ and began recording when the meter was deployed, and data were collected throughout the entire deployment. Current meters were installed on the OBEMs deployed at sites SH-R11 and QH-R10, and collected a total of approximately 11 days of data.

The OBEM records variations in both the Earth's natural-time MT field and the synthetic artificial source derived from the transmitter, as well as any noise generated by seawater motion. After instrument recovery, we downloaded the raw data from the OBEM data logger and current meter and calculated the five EM component power spectra using a short-time Fourier transformation (STFT) algorithm. We set the length 


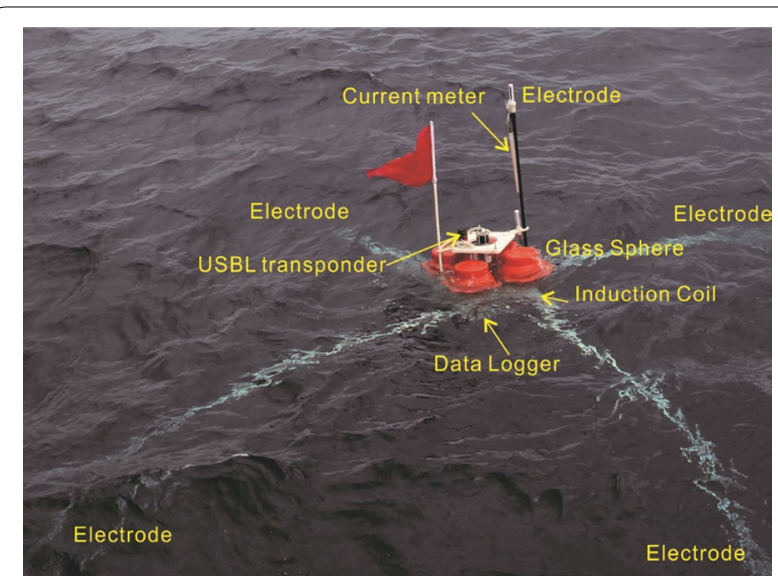

Fig. 2 An ocean bottom electromagnetic receiver (OBEM) recovery during the EM survey in the Shenhu area. The current meter is installed at the top of the vertical electrode arm, which floats freely via buoyant forces when the OBEM is deployed on the seafloor and measures current velocity using the tilt of the current meter

of the STFT to 4096 and the effective frequency band from approximately 0.05 to $75 \mathrm{~Hz}$. All of the raw time series were calculated using this method.

Figure 3 shows the seafloor current data and the five EM components STFT results from the SH-R11 site. The conductive seawater acts a low-pass filter on the natural MT signal, causing high-frequency band signals to decrease. We recorded two very noisy sections of data: one as the instrument fell to the seafloor, and another at the end of the deployment when the instrument floated to the surface. We observed four strong spectrum clusters located at $8 \mathrm{~Hz}$, as well as odd harmonic transmitted frequencies between 8-25 and 8-26 (i.e., the artificial CSEM signal). The first and second spikes are derived from the $8 \mathrm{~Hz}$ CSEM tow line, while the third and fourth spikes originated from the $0.5 \mathrm{~Hz}$ CSEM tow line. Except for the CSEM signal time section, there were several quiet periods during which the instrument was sitting on the seafloor.

At SH-R11, current speed peaks over $20 \mathrm{~cm} / \mathrm{s}$ are ten times what is expected in waters of comparable depth. We observed no 12- or 24-h period tidal modulations, but shorter period variations had a similar magnitude and there were long-term variations in speed that may have been modulated by tides. Noise existed throughout the entire deployment period, where approximately onethird of the data appear noisy. However, as observed from the E- and B-field power spectra, seafloor currents are characterized by a very rapid increase in magnitude on a very short timescale. The synchronous noise effect from the E- and B-fields can explain noise that is mainly due to seawater motion-induced magnetic fields. There is a strong correlation between EM noise and velocity: when velocity was faster than $2 \mathrm{~cm} / \mathrm{s}$, we observed clear EM noise signals in the data.

Figure 4 shows the relationship between current speed, induced EM noise, and the Earth's magnetic field. In the Shenhu area, the declination $(\delta)$ of Earth's magnetic field is approximately $10^{\circ}$, and the amplitude of the By component is small. The noise in the Ex component is larger than in the Ey component, which may explain why the current direction nearly couples orthogonally with the Ex component. Noise in the Ez component was the smallest.

Figure 5 illustrates a 172-s section of Fig. 3 that details the five EM field components and two direction current velocity components. Induced EM noise is too strong to identify any useful MT signal. Noise in the Ez component is smaller than noise in the Ey and Ex components. The eastern velocity component, $v_{\mathrm{E}}$, is larger than the northern component, $v_{\mathrm{N}}$. According to Fig. 4, which shows the directions between the velocity and EM component, the direction of Ex is $244^{\circ}$, derived from a heading sensor. Seawater motion-induced EM noise is orthogonal to velocity and couples with the EM component in the same direction. The corresponding velocity and EM field components are coherent, while a high-frequency disturbance is present. Coherent noise in the electric and magnetic fields, when present, lasts from a few minutes to as long as several hours, and have a $\sim 0.25 \mathrm{~Hz}$ frequency.

Figure 6 shows the power spectrum density results of the Qiongdongnan data (site QH-R10). Compared to the Shenhu area, Qiongdongnan has eight power spectrum clusters indicated in the CSEM signal time section. The first four spikes are the same as in the Shenhu area, where the fundamental transmission frequencies were $0.5 \mathrm{~Hz}$ and $8 \mathrm{~Hz}$. Two $8 \mathrm{~Hz}$ and two $0.5 \mathrm{~Hz}$ towed lines are visible around 9-5. Between 9-7 and 9-8, there are three CSEM time sections with a $0.125 \mathrm{~Hz}$ towed line and at 9-10, one time section with a $0.5 \mathrm{~Hz}$ towed line. The induced EM noise in the B-field is also larger than in the E-field. Maximum current speed is approximately $10 \mathrm{~cm} / \mathrm{s}$, which is lower than in the Shenhu area because the water is quieter in marine basin conditions than in shelf/slope conditions. There is a strong correlation between EM noise and velocity: when the velocity is larger than $2 \mathrm{~cm} / \mathrm{s}$, there is obvious EM noise in the B-field component and the noise in the E-field is smaller than in the B-field.

Figures 3 and 6 show that whenever current values are high, there are spectral maxima in at least two of the five measured EM components at the same frequency and at its harmonics. Figure 6 shows that such enhanced signals at this peak frequency can occur in the magnetic field, yet be absent from the electric components at the same time. The explanation for these effects is that the instruments are rocking, and it is their motion that matters for 

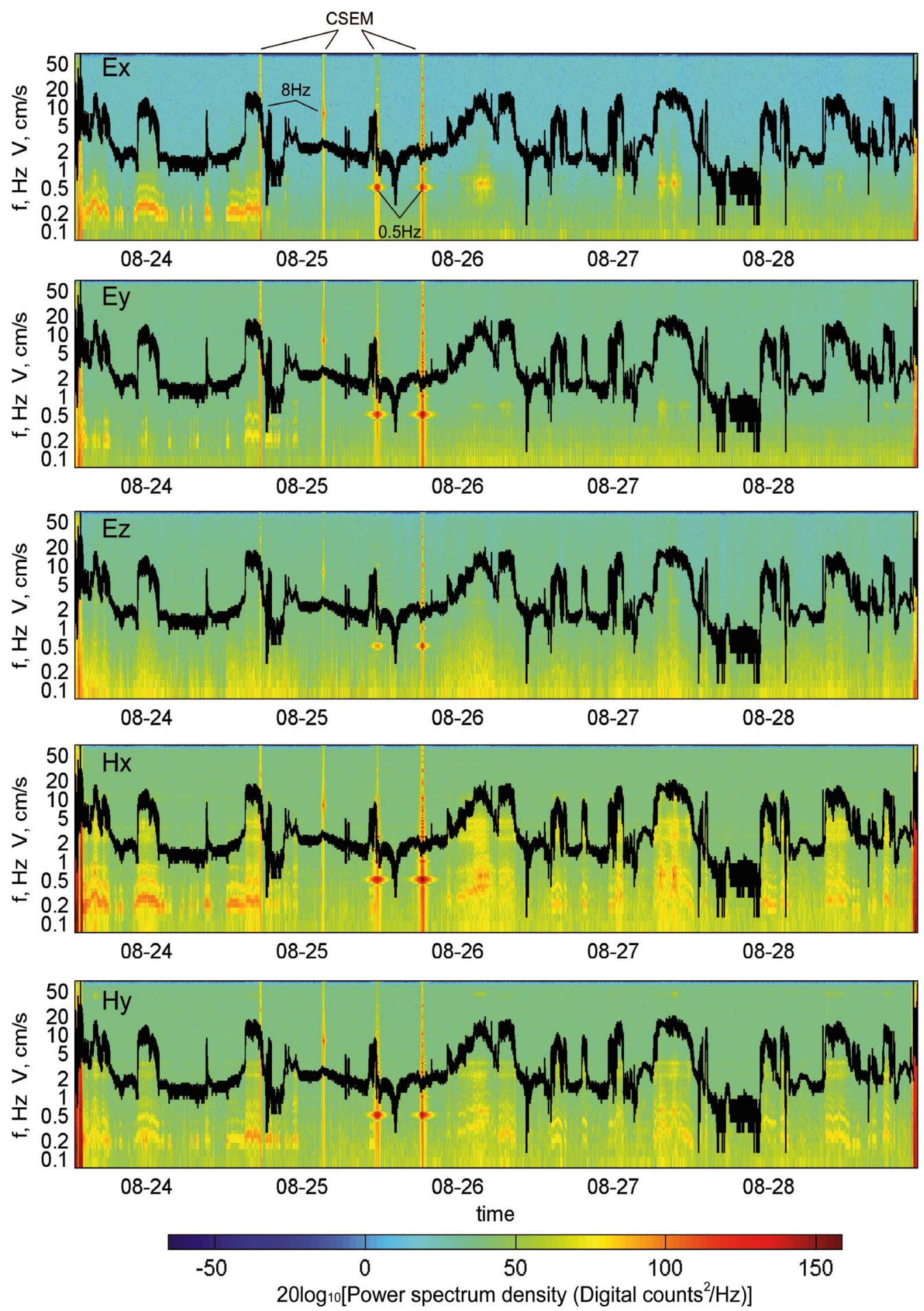

Fig. 3 Results from the short-time Fourier transformation (STFT) analysis of 5 days worth of five EM components and current speeds from site SH-R1 1 in the Shenhu area. The subplots are the Ex, Ey, Ez, Hx, and Hy components. Colors indicate the power spectrum density of the EM components. Units of the color bar are in $20 \log _{10}$ (digital counts ${ }^{2}$ per $\mathrm{Hz}$ ). The black line indicates current speeds in $\mathrm{cm} / \mathrm{s}$ 


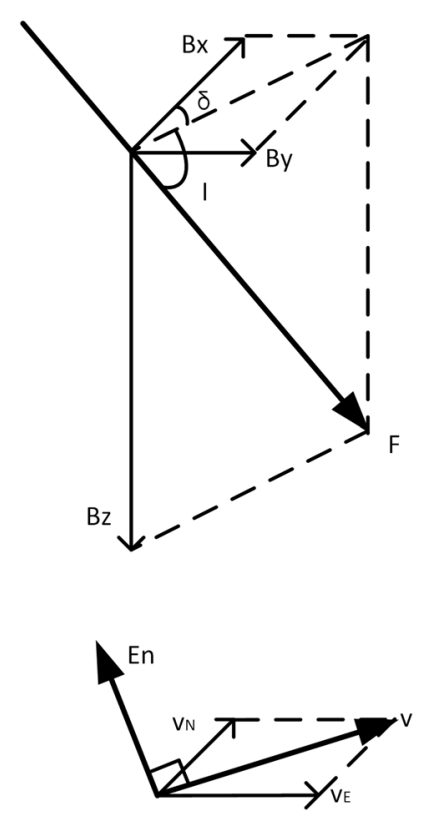

Fig. 4 A physical-mathematical model of induced EM noise from currents coupled with the Earth's magnetic field

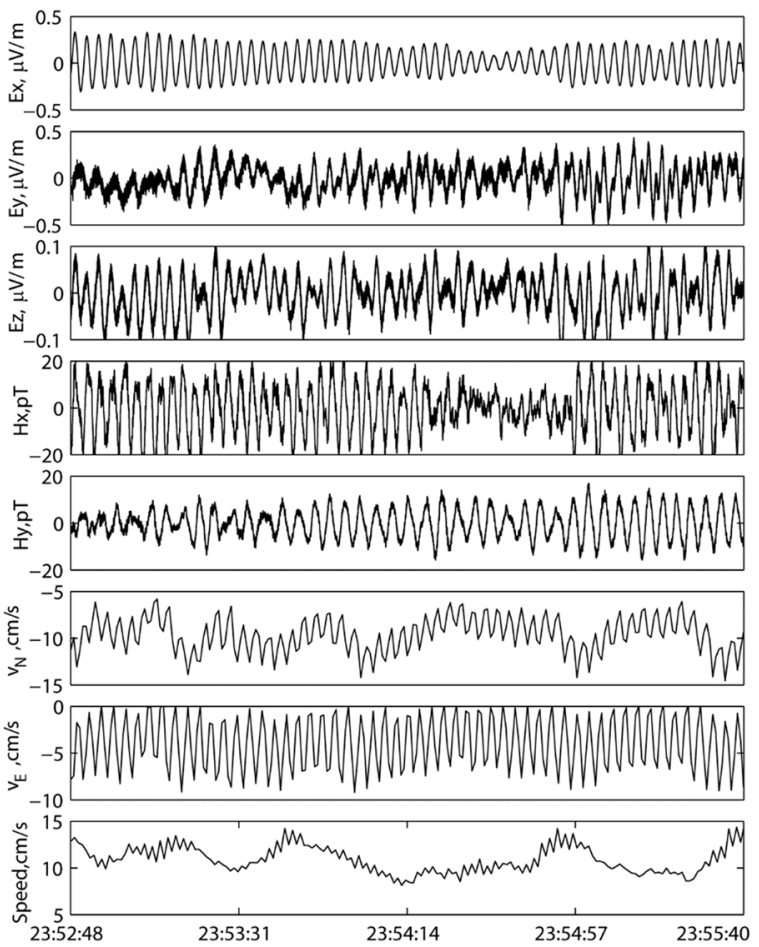

Fig. 5 A 172-s time series from site SH-R11 at August 24, 2018, when the velocity of the time section was relatively large. The five EM components (Ex/Ey/Ez/Hx/Hy) are derived from the OBEM data logger with a $150 \mathrm{~Hz}$ sampling rate, and the bottom three components ( $\left.v_{E} / v_{N} / S p e e d\right)$ originated from the current meter with a sampling rate of $1 \mathrm{~Hz}$ these characteristic frequencies. The EM noise source in Shenhu area was mainly caused by current-induced noise and instrument's rocking, while the EM noise source in Qiongdongnan was mainly caused by the instrument rocking.

\section{Adaptive correlation noise-canceling filter}

Because of inevitable and uncontrollable seawater motion, and because the velocity of seawater motion is highly variable over time, the electromagnetic noise caused by seafloor currents is also highly variable over time. Therefore, it is almost useless to use the frequency domain transfer function method to implicitly rely on a stationary assumption to remove noise. An adaptive filtering noise removal method for non-stationary timedomain data signals is therefore proposed. The most commonly used method is adaptive correlation noise removal (Haykin 2002). The adaptive correlation canceller combines the time-varying filter coefficients, which change in the best sense, and eliminates the unknown interference contained in the main signal by using a noise reference (where the main signal is weak). In our research, the primary polluted signal is a component of the magnetic field and the electric field, and the reference (noise) signal is the two horizontal components of current velocity. Each adaptive filter is a linear transverse finite impulse response (FIR) filter whose coefficients are updated by the least mean squares (LMS) algorithm at each time step.

In Fig. 7, $s(k)$ represents the EM component free noise, and added to a noise $n(k)$ is correlated in an unknown system with $v(k)$, which represents the current meter data. The signal polluted by noise is thus given by $d(k)$. At the discrete time $k$, let the predicted noise $y(k)$ be the outputs of the adaptive FIR filters. The input of the adaptive FIR filter is $v(k)$ and its adaptive coefficients, which are updated by the adaptive algorithm using the LMS algorithm.

Let the primary signal $d$ be given by:

$$
d=s+n,
$$

where $n$ represents the noise in $d$, and $s$ represents the noise-free signal. $n$ is correlated by an unknown system with the reference signal $v$ (the two seawater velocity components $v_{\mathrm{E}}$ and $v_{\mathrm{N}}$ ) (Fig. 7).

At the discrete time $k$, let the predicted noise $\mathbf{y}(k)$ be the sum of the outputs of the adaptive FIR filters, let the adaptive FIR filter coefficients be $W_{1}(k)$ and $W_{2}(k)$. The output of the adaptive FIR filter at time $k$ is:

$$
\mathbf{y}(k)=\mathbf{W}(k) \times \mathbf{v}(k)=W_{1}(k) \times v_{\mathrm{N}}(k)+W_{2}(k) \times v_{\mathrm{E}}(k),
$$



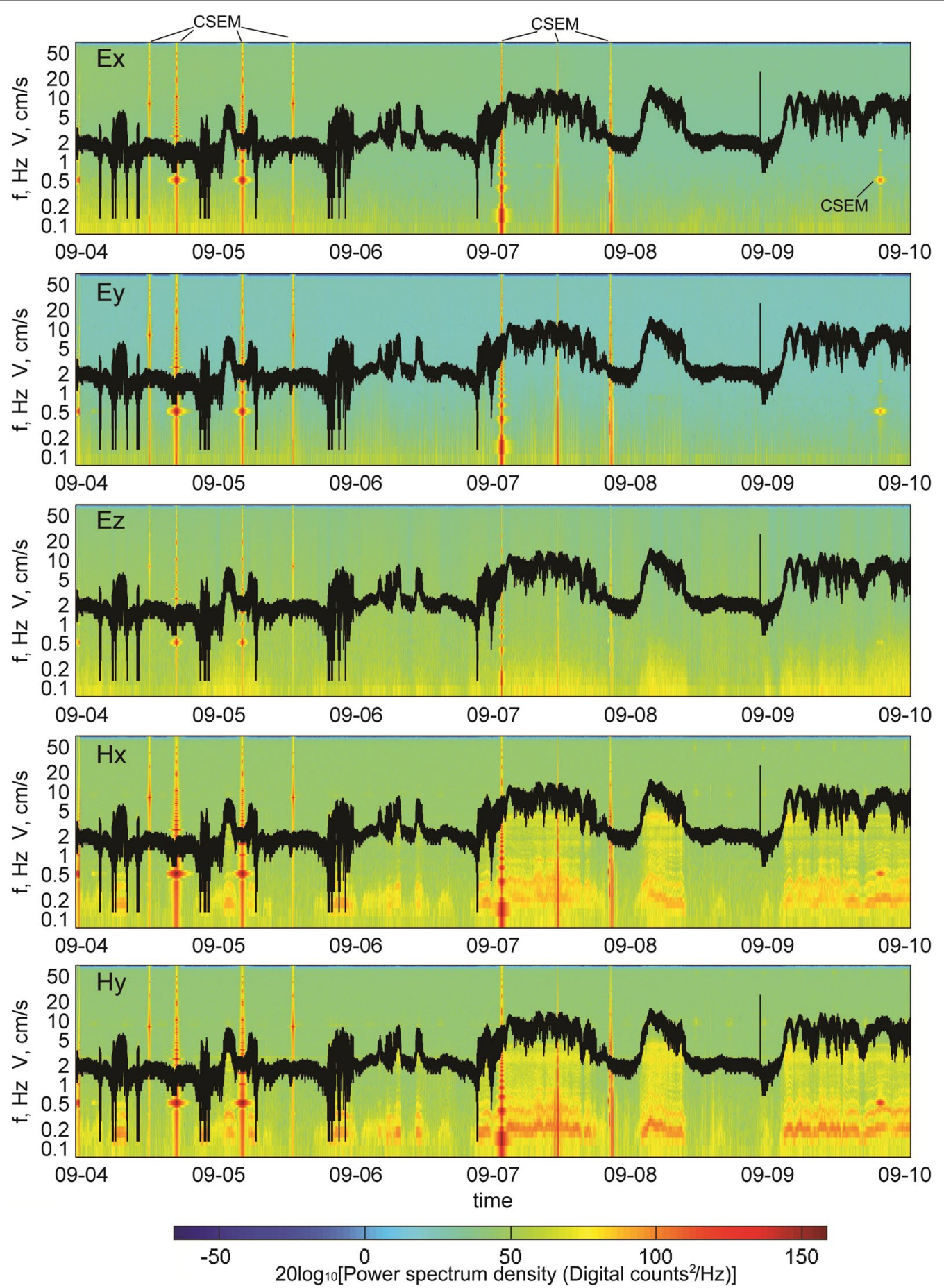

Fig. 6 Short-time fourier transform results of the five EM components and current speeds for 6 days at site QH-R10 in the Qiongdongnan area. Colors indicate the power spectrum density of the EM components. Units of the color bar are in $20 \log _{10}$ (digital counts ${ }^{2}$ per $\mathrm{Hz}$ ). The black line indicates the current speed in $\mathrm{cm} / \mathrm{s}$ 


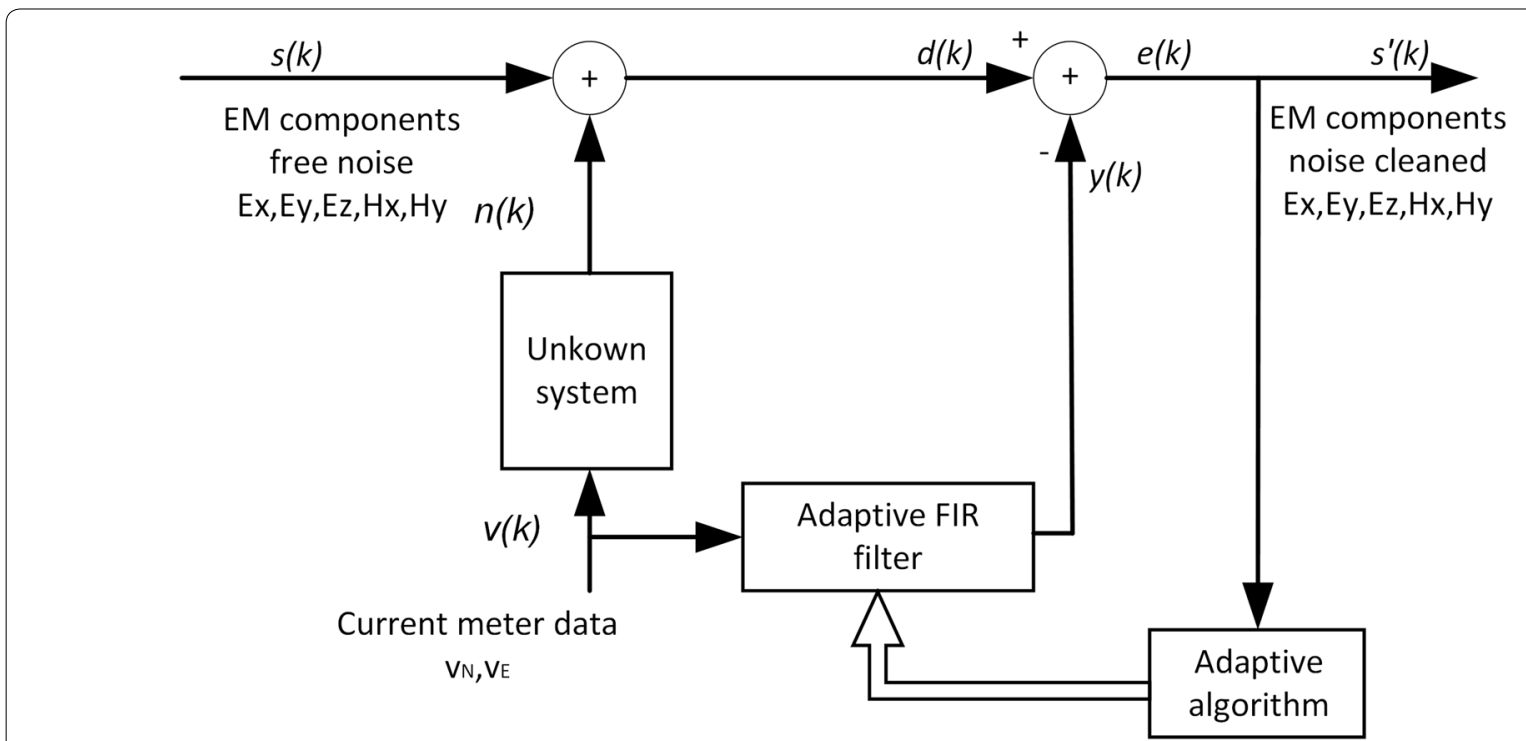

Fig. 7 Diagram of the adaptive correlation cancellation filter developed to cancel seawater motion-induced EM noise. The $s(k)$ represents the EM component free noise, and $s^{\prime}(k)$ represents the residual uncorrelated output signal, which is the cleaned noise

and the error signal $e(k)$ is defined as difference between the primary signal $d(k)$ and the filter output signal $y(k)$ :

$$
e(k)=d(k)-y(k) \text {. }
$$

Using the LMS algorithm, at time step $k+1$ the filter (the matrix $\mathbf{W}$ in Eq. 2) is given by:

$$
\mathbf{W}(k+1)=\mathbf{W}(k)+\frac{\mu \times \mathbf{e}(k) \times \mathbf{v}(k)^{t}}{\alpha+\sum_{i=1}^{N} \mathbf{v}(k-i)^{2}},
$$

with the superscript $t$ representing the transpose of the matrix $\mathbf{v}$ and $N$ representing the filter length. The coefficient $\mu$ is the adaption step size of the time-domain filter and $\alpha$ is a damping factor. For further steps, $\mathbf{W}$ will start from the previous result, computing the following $\mathbf{W}(k)$ as in Eq. 4 to result in the output $\mathbf{e}(k)$ (Eq. 3) from the sum of the predicted noise (Eq. 2) over the filter length $N$ :

$$
\mathbf{y}(k)=\sum_{i=1}^{N} \mathbf{W}(k-i) \times \mathbf{v}(k-i) .
$$

The adaptive correlation noise-canceling filter applied to the SH-R11 raw data gave generally improved results. Figure 8 illustrates segments of the noise-canceled EM component signals from the raw noise-influenced time series from site SH-R11. The adaptive correlation filter achieved major reductions in the peak signal amplitude, thus reflecting an obvious reduction of the induced EM noise. E-field and B-field time series of noise-canceled

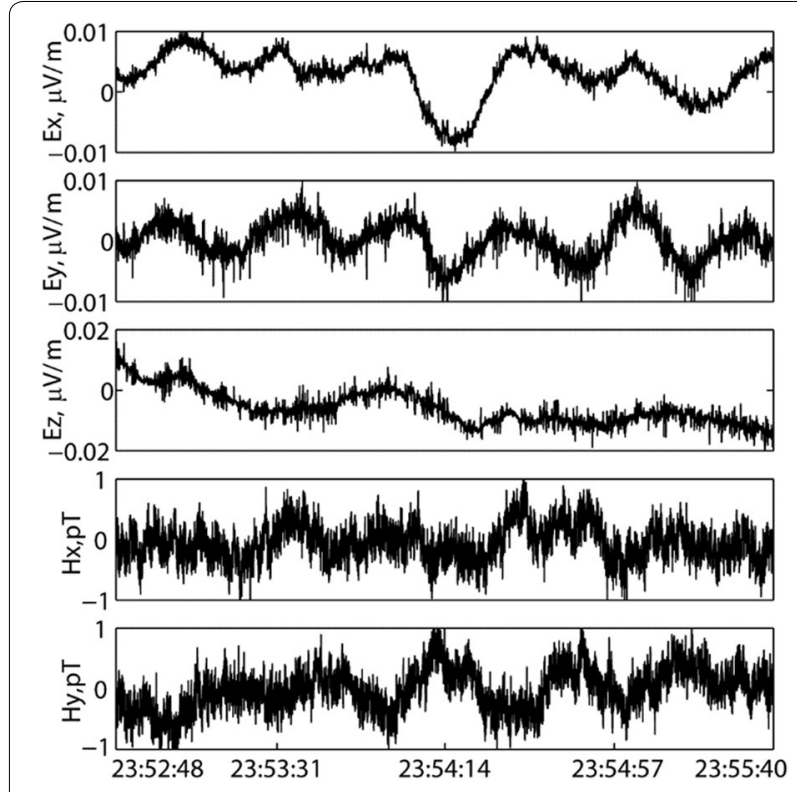

Fig. 8 Noise-canceled time series windows of five component EM fields from the SH-R1 1 site, shown in Fig. 5. The five plots from top to bottom illustrate the five EM components without noise

segments demonstrate this, with amplitudes one to two orders of magnitude below the peak amplitude of a noise-influenced segment (Fig. 5). When the velocity is lower than a pre-defined threshold (in this example, the threshold was set as $2 \mathrm{~cm} / \mathrm{s}$ ), the EM noise is quiet and the filter will not work. 


\section{MT data-processing results}

The newly developed method was applied to process MT time series collected in both the Shenhu and Qiongdongnan areas. These time series were strongly "polluted" by instrument rocking and induced EM noise caused by seafloor currents, which made one-third of the time series unusable for subsequent data processing under traditional MT response function estimation methods. We chose data from stations in the Shenhu area to demonstrate the time series separation method below. The water depth is approximately $1500 \mathrm{~m}$, and high-frequency band signals are strongly attenuated and it is impossible to estimate the MT response function for periods under $10 \mathrm{~s}$ due to the low signal-to-noise ratio. Figure 9 shows the apparent resistivity and phase at four sites (SH-R8, SH-R10, SH-R11, and SH-R13). Seafloor current-induced EM noise occurred at all four sites, and the data were contaminated at different levels.

We applied the above-mentioned adaptive correlation filter to reduce the induced EM noise at site SH-R11.
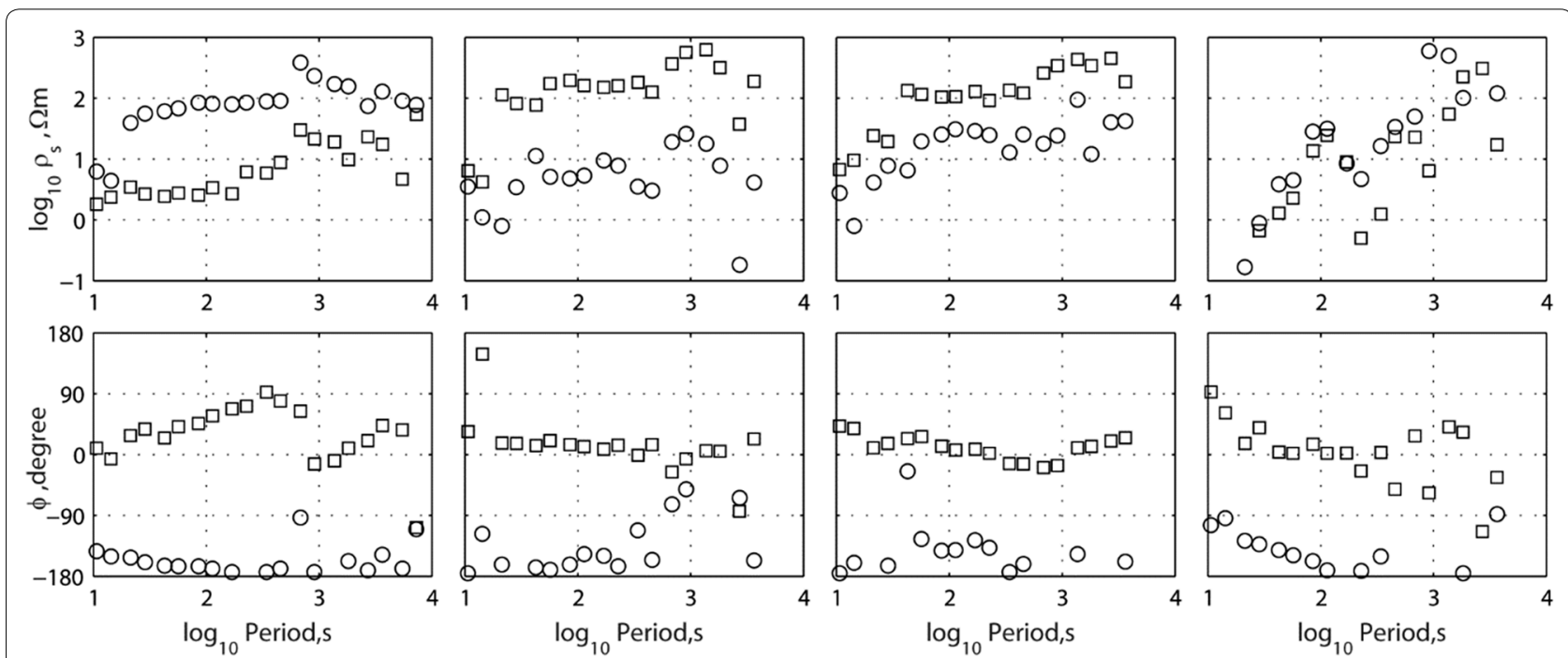

Fig. 9 Marine MT processing results, which are raw data and not pre-filtered by the adaptive canceling filter. From left to right: the resistivity and phases of site SH-R8, SH-R10, SH-R11, and SH-R13 are shown. The xy components are represented by squares, and the yx components are represented by circles
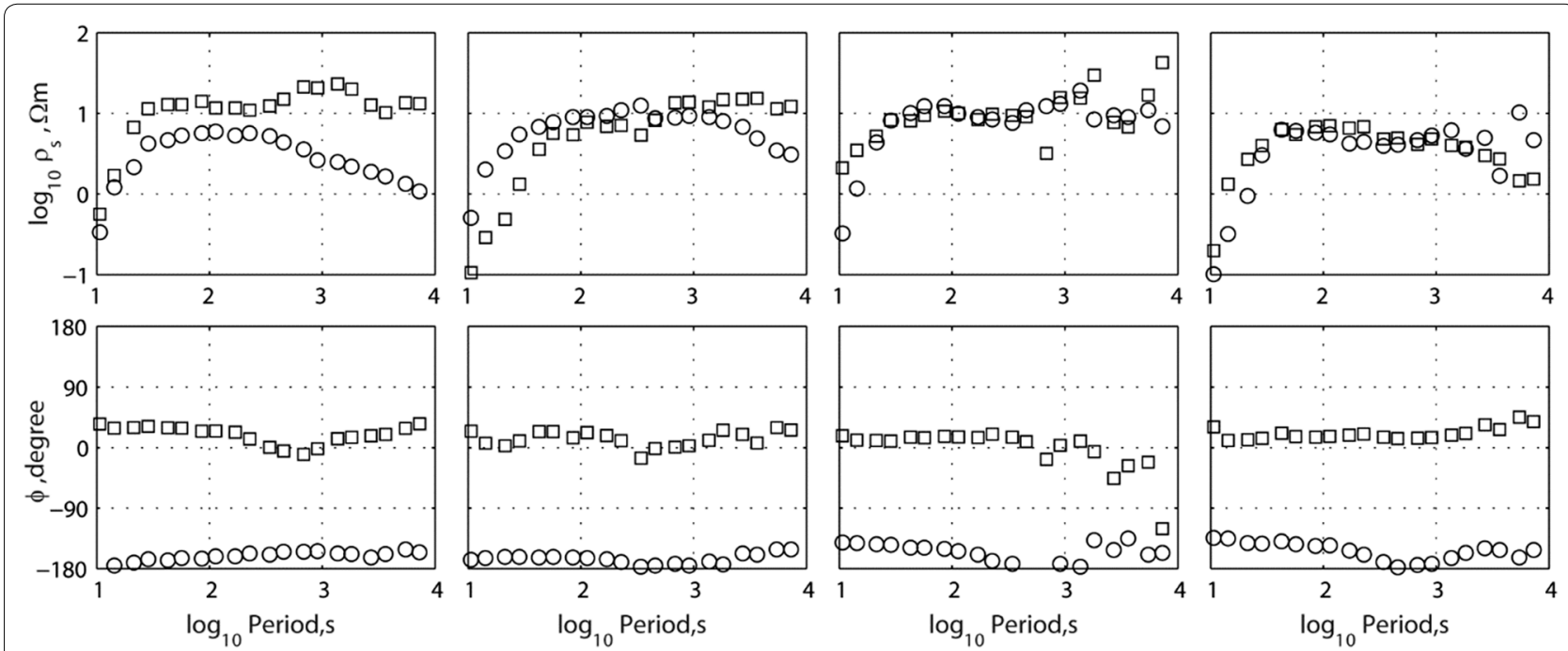

Fig. 10 Results of raw MT data processing, pre-filtered with the adaptive cancellation filter. From left to right, plots show resistivity and phase curves from sites SH-R8, SH-R10, SH-R11, and SH-R13. The xy and yx components are denoted by squares and circles, respectively 
MT impedances estimated from the raw time series after applying the adaptive correlation noise-canceling filter showed obvious improvements over the estimates compared to results obtained without the filter. Figure 10 shows the MT impedances for the above four estimates from the unfiltered and filtered time series, showing the improvement between both apparent resistivity and phase at frequencies lower than $0.1 \mathrm{~Hz}$.

To improve data quality over the entire frequency range for other sites, nearby reference processing by setting up the current data as a nearby reference site for other sites in the Shenhu area was applied, up to approximately $1 \mathrm{~km}$ from the current meter measurement site (SH-R11). The filter successfully improved the MT impedance of both local sites and other nearby sites.

The data were processed using the previously mentioned adaptive correlation noise-canceling filter. Finally, the MT impedances were estimated using the SSMT2000 software package from Phoenix Geophysics. The MT impedances were computed for each frequency band, and the apparent resistivity and the phase curves for the entire frequency range were all obtained.

The data quality was obviously improved and is comparable to the results obtained without the above-mentioned filter. Even though further work is still required, these preliminary data-processing results demonstrate that utilizing adaptive correlation noise-cancellation filters with current data is an effective method for MT data processing.

\section{Discussion and conclusions}

Depending on offshore conditions, marine MT signals are "polluted" by seafloor current-induced EM noise at varying levels. The raw MT time series from the continental shelf in the Shenhu area shows such strongly correlated noise that we could not obtain useable impedance estimates using traditional data-processing techniques. The basin stations in the Qiongdongnan area show much less current-related EM noise and have longer quiet segments in the data than in the Shenhu area. The maximum current speeds in Shenhu and Qiongdongnan were approximately $20 \mathrm{~cm} / \mathrm{s}$ and $10 \mathrm{~cm} / \mathrm{s}$, respectively, and EM noise was apparent whenever the current speed was faster than $2 \mathrm{~cm} / \mathrm{s}$.

In this paper, we show that an adaptive correlation noise-cancellation filter is an effective method for MT data processing and can be used to reduce induced EM noise by using local current data. Therefore, this method is applicable to datasets with co-located current data, and has the potential to be an effective filter for MT data processing. The adaptive correlation noise-cancellation filter improved data quality significantly when the data had been contaminated by seawater motion-induced noise.
However, installing a current meter on each OBEM would increase the cost for marine MT data acquisition. One current meter data used for multiple nearby sites may be more feasible. The effective distance of local and distal reference sites may be decided by the complexity of currents in a particular study area.

In the future, we will combine the adaptive correlation noise-cancellation filter with robust processing procedures. In addition, adaptive correlation noise-cancellation filters may have the potential to separate noise from raw time series for marine MT data processing, where the MT signal is often contaminated by strong noise caused by induced EM noise due to seawater motions.

\section{Abbreviations}

CSEM: controlled source electromagnetic; EM: electromagnetic; LMS: leastmean-square; MT: magnetotelluric; OBEM: ocean bottom electromagnetic receiver; RMEV: robust multivariate errors-in-variables; STFT: short-time Fourier transform

\section{Acknowledgements}

Thanks for the extensive support offered by the respective captains, the crew of the ship, and the marine technicians aboard the R/V "Haiyangsihao". Professor Xia gave some suggestions on adaptive canceling filters. Professor Wu provided valuable comments regarding data processing. We particularly thank Drs. Zhang and Tu for their helpful suggestions and support on the current meter. The SSMT2000 processing software was provided by Phoenix Geophysics Ltd. Comments provided by the reviewers helped to improve the clarity of the manuscript.

\section{Authors' contributions}

KC developed the required hardware and software, led the overall design, and performed the offshore experiments. MD was the noise reduction technology consultant. QZ and XL assisted with the marine EM survey. JJ participated in the MT data processing. All authors read and approved the final manuscript.

\section{Funding}

The general study and experiment were funded by the National Key R\&D Program of China (2016YFC0303100, 2017YFF0105700). The development of the OBEM was supported by the National Science Foundation of China (41804071, 61531001).

\section{Availability of data and materials}

(1) Partial raw time series of EM components data. (2) Partial raw time series of current meter data.

\section{Competing interests}

The authors declare that they have no competing interests.

\section{Author details}

1 School of Geophysics and Information Technology, China University of Geosciences, Beijing, China. ${ }^{2}$ Institute of Marine Geological Exploration Method and Technology, Guangzhou Marine Geology Survey, Guangzhou, China.

Received: 11 February 2019 Accepted: 26 November 2019

Published online: 13 January 2020

\section{References}

Baba K, Chave AD, Evans RL, Hirth G, Mackie RL (2006) Mantle dynamics beneath the East Pacific Rise at 17 degrees S: insights from the Mantle Electromagnetic and Tomography (MELT) experiment. J Geophys Res Solid Earth. https://doi.org/10.1029/2004jb003598 
Chave AD, Luther DS (1990) Low-frequency, motionally induced electromagnetic fields in the ocean: 1. Theory. J Geophys Res Oceans 95(C5):71857200. https://doi.org/10.1029/JC095iC05p07185

Constable S, Heinson G (2004) Hawaiian hot-spot swell structure from seafloor MT sounding. Tectonophysics 389(1-2):111-124. https://doi. org/10.1016/j.tecto.2004.07.060

Constable SC, Orange AS, Hoversten GM, Morrison HF (1998) Marine magnetotellurics for petroleum exploration. Part I: a sea-floor equipment system. Geophysics 63(3):816-825. https://doi.org/10.1190/1.1444393

Egbert GD (1997) Robust multiple-station magnetotelluric data processing. Geophys J Int 130(2):475-496. https://doi.org/10.1111/j.1365-246X.1997. tb05663.x

Filloux JH (1980) North pacific magnetotelluric experiments. Earth Planets Space 32:SI33-SI43. https://doi.org/10.5636/jgg.32.Supplement1_SI33

Haykin SS (2002) Adaptive filter theory, 4th edn. Prentice Hall, New Jersey

JianEn J, QingXian Z, Ming D, XianHu L, Kai C, Meng W, GuangHong T (2018)

A study on natural gas hydrates and their forming model using marine controlled-source electromagnetic survey in the Qiongdongnan Basin. Chin J Geophys 61(11):4677-4689. https://doi.org/10.6038/cjg2018L06 60 (in Chinese)

Kai C, Wenbo W, Ming D, Zhongliang W, Gang Y (2015) A new marine controlled-source electromagnetic receiver with an acoustic telemetry modem and arm-folding mechanism. Geophys Prospect 63(6):14201429. https://doi.org/10.1111/1365-2478.12297

Key K, Constable S (2002) Broadband marine MT exploration of the East Pacific Rise at 950'N. Geophys Res Lett 29(22):2054-2057
Key KW, Constable SC, Weiss CJ (2006) Mapping 3D salt using the 2D marine magnetotelluric method: case study from Gemini Prospect, Gulf of Mexico. Geophysics 71(1):B17-B27. https://doi.org/10.1190/1.2168007

Lezaeta PF, Chave AD, Evans RL (2005) Correction of shallow-water electromagnetic data for noise induced by instrument motion. Geophysics 70(5):G127-G133

Naif S, Key K, Constable S, Evans RL (2015) Water-rich bending faults at the Middle America Trench. Geochem Geophys Geosyst 16(8):2582-2597. https://doi.org/10.1002/2015GC005927

Neska A, Nowozynski K, Reda J, Jegen-Kulcsar M (2013) Reducing motion noise in marine magnetotelluric measurements by means of tilt records. Geophys J Int 194(1):304-315

Sanford TB (1971) Motionally induced electric and magnetic fields in the sea. J Geophys Res 76(15):3476-3492

Vozoff K (1972) The magnetotelluric method in the exploration of sedimentary basins. Geophysics 37(1):98-141. https://doi.org/10.1190/1.1440255

Wang M, Deng M, Wu Z, Luo X, Jing J, Chen K (2017) The deep-tow marine controlled-source electromagnetic transmitter system for gas hydrate exploration. J Appl Geophys 137:138-144

\section{Publisher's Note}

Springer Nature remains neutral with regard to jurisdictional claims in published maps and institutional affiliations.

\section{Submit your manuscript to a SpringerOpen ${ }^{\circ}$ journal and benefit from:}

- Convenient online submission

- Rigorous peer review

- Open access: articles freely available online

- High visibility within the field

- Retaining the copyright to your article

Submit your next manuscript at springeropen.com 